\title{
Una mirada a la alternancia de pares incoativo-causativo en $k^{\prime} i c h e^{\prime 1,2}$
}

\section{A Glance at Inchoative-Causative Alternation Pairs in K'iche'}

Telma Angelina Can Pixabaj

CIMSUR-UNAM, email: kaantelma@gmail.com

Resumen:

En este estudio se describe y analiza la alternancia de pares incoativo-causativo en k'iche' siguiendo las propuestas tipológicas de Haspelmath (1993). Ese autor indica que los verbos en la alternancia remiten a pares de verbos que expresan la misma situación y difieren en que el causativo incluye un causante externo, mientras que el incoativo no; el autor toma como base datos de 21 lenguas, de las cuales ninguna es mesoamericana. Los resultados de este análisis aportan, por un lado, datos relevantes al estudio tipológico del tema, por ejemplo, la ocurrencia del anticausativo, que no es común en muchas lenguas, pero que el k'iche' sí lo registra; y por otro lado, pueden ser generalizados a otras lenguas mayas, en algunos aspectos como el uso de los posicionales y de verbos con morfología pasiva y antipasiva para formar el incoativo.

Palabras claves: posicionales, pasivo y antipasivo, alternancia de verbos.

\section{Abstract:}

Following Haspelmath's typological proposals (1993), this study describes and analyzes pairs of inchoative-causative alternation in the K'iche' language. The author indicates that the verbs in alternation remit to pairs of verbs expressive of the same situation and differ inasmuch as the causative aspect includes an external cause, whereas the inchoative does not. The author uses data from 21 languages, none of which is Mesoamerican. On one hand, the results of this analysis contribute relevant data to a typological study of the topic, such as the occurrence of the anticausative form, which is not common in many languages, but is recorded in the $K^{\prime}$ iche' language. On

1 Una versión preliminar de este artículo fue presentada en el XII Encuentro Internacional de Lingüística en el Noroeste, Hermosillo 2012. Se ha enriquecido gracias a los comentarios y sugerencias recibidos durante dicho encuentro. También me he beneficiado de opiniones de Nora England, Roberto Zavala, B'alam Mateo Toledo y otras personas a quienes les expreso un profundo agradecimiento. Asimismo, manifiesto mi gratitud a los dictaminadores de este artículo porque sus observaciones me han ayudado a mejorarlo. Todos los errores son mi responsabilidad.

2 El k'iche' es un idioma maya utilizado en Guatemala por alrededor de un millón de hablantes (Richards 2003). Se encuentra disperso geográficamente en 76 municipios de nueve departamentos, principalmente del altiplano del país. El k'iche' es robusto en términos del número de hablantes; y a pesar de ello y de los proyectos y programas para su mantenimiento, se observa su desplazamiento acelerado por el español en muchos contextos. Como consecuencia, las nuevas generaciones ya no están aprendiéndolo. Aunque es uno de los idiomas mayas más estudiados, existen temas o fenómenos que requieren una mayor profundización. 
the other hand, this could be generalized to other Mayan languages, specifically in some aspects, such as the use of positional verbs and verbs with a passive and antipassive morphology in order to form the inchoative.

Keywords: positional verbs, passive and antipassive morphology, verb in alternations.

Recibido: 30/09/2016

Aceptado: 7/11/2016

\section{Introducción}

Según Haspelmath (1993: 90), la alternancia de pares incoativo-causativo se refiere a pares de verbos que expresan la misma situación semántica y difieren en que el causativo incluye un causante externo, como en (1a) del español, mientras que el incoativo no, como en (1b).
(1) a. matar (verbo transitivo)
b. morir (verbo intransitivo)

En cuanto a forma, en (1) se puede observar que cada verbo tiene un origen y una forma diferentes. Es decir, uno no depende o no se deriva del otro. Sin embargo, puede haber lenguas o casos en donde una forma se derive de la otra, como el ejemplo ruso en (2). En (2) la forma básica es el verbo causativo transitivo en (a) y la forma derivada es el verbo incoativo intransitivo en (b) que se forma por medio de un sufijo.
(2) a. rasplavit
'derretirlo' (transitivo)
b. rasplavit-sja
'derretirse' (intransitivo)
(Haspelmath 1993:89)

En otros casos, un mismo verbo se utiliza para indicar el par incoativo-causativo. El ejemplo en (3) es del inglés y muestra que el verbo 'break' (Haspelmath 1993:90) funciona tanto para la forma incoativa intransitiva en (a) como para la causativa transitiva en (b).
(3) a. The stick broke
'El palo se quebró.'
(forma intransitiva)
b. The girl broke the stick 'La muchacha quebró el palo' (forma transitiva) 
Con base en los ejemplos se puede decir que no hay un solo patrón de codificación de la alternancia de pares incoativo-causativo. Al respecto, Haspelmath (1993) presenta tres patrones de codificación: causativo, anticausativo, como el ejemplo (2), y no directo, en donde hay tres subtipos (equipolente, lábil y supletivo) los cuales incluyen el ejemplo en (1) y el (3). Es decir, en muchos casos hay una forma básica y una forma derivada, pero este no ocurre siempre así. Los detalles se presentan en $\S 3.1$.

Dichos patrones se pueden encontrar en k'iche': el anticausativo (4-5), el causativo (6) y únicamente se registra la subclase equipolente (7) para la clase no directa.

\section{Anticausativo}

(4)
a. $x-\varnothing-u-k^{\prime} a t-o$
$\rightarrow$
b. $x-\varnothing-k^{\prime}$ aat-ik ${ }^{3}$
CP-B3SG-A3SG-quemar-SC
CP-B3SG-quemar. PAS-SC
'lo quemó'
'se quemó'

(5)
a. x-ø-u-paq'ii-
$\rightarrow$
b. $x-\varnothing-p a q^{\prime} i-\mathbf{n}-i k^{4}$
CP-B3SG-A3SG-explotar-ACT
CP-B3SG-explotar-AP-SC
'lo explotó'
'(se) explotó'

Causativo
(6) a. x-ø-saq-ir-ik
$\rightarrow$
b. x-ø-u-saq-ir-saa-j
CP-B3SG-blanco-VI-SC
CP-B3SG-A3SG-blanco-VI-CAU-SC
'se puso blanco'
'lo emblanqueció'

Equipolente
(7) a. $x-\varnothing-t^{\prime} u y-e^{\prime}-i k$
b. x-ø-u-t'uy-ub'aa'
CP-B3SG-sentar-VI-SC
CP-B3SG-A3SG-sentar-VT
'se sentó'
'lo sentó'

3 Las abreviaturas empleadas son: $\mathrm{A}=$ ergativo (JA), AGT = agentivo, AP = antipasivo, $\mathrm{B}=$ absolutivo (JB), $C A U$ = causativo, $C P=$ completivo, $D E T=$ determinante, $\mathrm{DIR}=$ direccional, $\mathrm{EST}=$ estativo, $\mathrm{FOC}=$ foco, IMP = imperativo, NEG = negación, $\mathrm{PAC}=$ paciente, $\mathrm{PAS}=$ pasivo, $\mathrm{PL}=$ plural, $\mathrm{SC}=$ sufijo de categoría, $S G=$ singular, $S R=$ sustantivo relacional, $V E=$ vocal epentética, $\mathrm{VI}=$ verbo intransitivo, $\mathrm{VT}=$ verbo transitivo

${ }^{4}$ Los verbos transitivos derivados tienen vocal prolongada en la última sílaba cuando el verbo se encuentra en posición final de la frase entonacional y la vocal es corta cuando el verbo no se encuentra en dicha posición o cuando la base recibe otros sufijos, como en (4a). 
El objetivo de este estudio es presentar una descripción y un análisis de la alternancia de pares incoativo-causativo en k'iche' siguiendo las propuestas tipológicas de Haspelmath (1993). Para ello voy a mostrar que en k'iche' existen los tres patrones de codificación que Haspelmath indica (anticausativo, causativo y no directo, aunque de este último solo hay un subtipo, el equipolente, como mostraré más adelante). Bajo la discusión de cada tipo de alternancia indicaré las clases de raíces o bases que conforman las formas básicas y cómo se construyen las formas derivadas.

En cuanto al tipo anticausativo, la forma incoativa se deriva con los mismos morfemas que se usan para formar los pasivos, como en (4b) y antipasivos, como en (5b); más adelante muestro la distribución que tienen estos morfemas. En cuanto al tipo causativo, este se deriva principalmente con el causativo morfológico -(ti)saa, como en (6). Finalmente, el tipo equipolente tiene una raíz posicional como base tanto para la formación del incoativo como para la formación del causativo, como en (7).

Cabe resaltar que el uso de la morfología pasiva y antipasiva para la formación del par incoativo en el patrón anticausativo, así como el uso de una raíz posicional para la base del equipolente son aspectos que no se han tratado con atención en la gramática del k'iche', al igual que en otras lenguas mayas. De manera que, aunque este trabajo no es de tipo comparativo, se puede decir que los resultados pueden ser generalizados a otras lenguas mayas, especialmente el uso de raíces posicionales y derivaciones pasivas y antipasivas en la alternancia incoativo-causativo. Se espera que los resultados sean una contribución al estudio tipológico de este tema.

Los temas que voy a cubrir son los siguientes:

i) Los patrones de codificación o tipos de pares de alternancia que existen en $k^{\prime} i c h e^{\prime}$ y las clases de palabras que participan en ellos. Algo muy interesante es que en 'k'iche' una de las clases viene de raíces posicionales y las otras, en cambio, pueden venir de verbos $u$ otras clases de palabras.

ii) La morfología que se emplea para las formas derivadas en los tipos de alternancia. Algunas formas derivadas, por ejemplo, tienen morfología pasiva y antipasiva.

iii) Finalmente, la definición del par incoativo de la alternancia resalta que este verbo no tiene un causante externo. Para mostrar esto en k'iche' voy a recurrir al uso de un agente oblicuo en los casos en que la forma incoativa tiene morfología pasiva. Para los incoativos con forma antipasiva voy a usar un paciente oblicuo 
para mostrar que al agregarlo cambia la relación del sujeto con respecto al verbo incoativo y ya no incluye la noción de incoativo.

Este artículo se organiza en tres partes. En § 2 expongo nociones gramaticales básicas sobre el k'iche' para la inteligencia del tema, los datos y la metodología. En $\S 3$ presento los datos que nos ocupan, descripción y análisis de la alternancia de pares incoativo-causativo en k'iche'. En $\S 4$ hago un resumen de los datos y en $\S 5$ presento algunas conclusiones.

\section{Nociones gramaticales básicas sobre el k'iche'}

Los datos son principalmente del municipio de Santa Lucía Utatlán (SL), del departamento de Sololá.

\subsection{Rasgos generales y tipológicos del k'iche'}

El k'iche' tiene marcación en el núcleo y tiene orden vos al igual que la mayoría de las lenguas mayas. Es una lengua ergativa (morfológica y sintácticamente, aunque en este artículo no se mostrarán los detalles), marca de igual forma el s (sujeto intransitivo) y o (objeto transitivo) con marcas absolutivas, también marca de manera diferente al A (sujeto transitivo) con marcas ergativas. Estos dos juegos de marcas de persona se denominan B y A, respectivamente, en la literatura maya. En la Tabla 1 muestro estos marcadores de persona.

Tabla 1. Marcadores de juego B y juego A

\begin{tabular}{|c|c|c|c|c|}
\cline { 3 - 5 } & \multicolumn{2}{c}{} & \multicolumn{2}{c|}{ Ergativo juego A } \\
\hline Número & Persona & Absolutivo, juego B & Prevocálico & Preconsonántico \\
\hline \multirow{3}{*}{ Singular } & $1^{\text {ra }}$ & in- & $w-i n w-$ & $n u-$ \\
\cline { 2 - 5 } & $2^{\text {da }}$ & $a t-$ & $a w-$ & $a-$ \\
\cline { 2 - 5 } & $2^{\text {da } F}$ & $=l a^{*}$ & $=l a$ & $=l a$ \\
\cline { 2 - 5 } & $3^{\text {ra }}$ & $\varnothing$ & $r-$ & $u-$ \\
\hline
\end{tabular}




\begin{tabular}{|c|c|c|c|c|}
\hline \multicolumn{2}{c|}{} & \multicolumn{2}{c|}{} & \multicolumn{2}{c|}{ Ergativo juego A } \\
\hline \multirow{2}{*}{ Número } & Persona & Absolutivo, juego B & Prevocálico & Preconsonántico \\
\hline \multirow{2}{*}{ Plural } & $1^{\text {ra }}$ & oj- & $q-$ & $q a-$ \\
\cline { 2 - 5 } & $2^{\text {da }}$ & $i x-$ & $i w-$ & $i-$ \\
\cline { 2 - 5 } & $2^{\text {da }} \mathrm{F}$ & $=a l a q$ & $=a l a q$ & $=a l a q$ \\
\cline { 2 - 5 } & $3^{\text {ra }}$ & ee-, e- & $k-$ & $k i-$ \\
\hline
\end{tabular}

* La segunda personal formal u honorífica generalmente se realiza como enclítico, así como se muestra en la Tabla 1. Puede ser proclítico, singular lal o plural alaq, cuando funciona como el sujeto de un predicado no verbal. Cuando funciona como sujeto de un verbo intransitivo, puede ser proclítico solo si es plural, pero no cuando es singular. Y cuando el verbo es transitivo, la segunda personal formal, singular o plural no puede ser proclítico.

Fuente: elaboración propia

En k'iche' se distinguen varias clases de palabras de las cuales solo voy a indicar las que sirven como raíces o bases para formar los verbos que participan en la alternancia incoativo-causativo. Las palabras de las que se derivan dichos verbos pueden ser: adjetivos, sustantivos y posicionales, además de los verbos mismos. Abajo indico la forma de cada clase de palabra.

\subsection{Sustantivos y adjetivos}

Los adjetivos pueden funcionar como atributivos o modificadores nominales, como en (8a) y como núcleos predicativos, como en (8b).

(8) a. x-ø-ch'aq-e' le jun chaqi'j-a chee' CP-B3SG-mojar-VI DET uno seco-EPEN árbol 'ese árbol seco se mojó'
b. øsaq le kotz'i'j
B3SG blanco DET flor

'la flor es blanca'

Los adjetivos se verbalizan principalmente por medio del sufijo - $V r$, como se muestra en (9) y se convierten en verbos intransitivos incoativos. 
(9) a. x-ø-chaqi'j-ar-ik

CP-B3SG-Seco-VI-SC

'se secó'

b. $x-\varnothing$-saq-ir-ik

CP-B3SG-blanco-VI-SC

'se puso blanco'

Ya convertidos los adjetivos en verbos intransitivos se les puede agregar el causativo -(ti)saa para convertirlos en verbos transitivos, como en (10).

(10) a. x- $\varnothing$-u-chaqi'j-ar-saa-j

CP-B3SG-A3SG-Seco-VI-CAU-ACT

'lo secó'

b. $\mathrm{x}$ - $\varnothing$-u-saq-ir-saa-j

CP-B3SG-A3SG-blanco-VI-CAU-ACT

'lo puso blanco'

Los sustantivos pueden funcionar como argumentos oracionales ( $\mathrm{A}$, S y O), como en (11a-c) y como núcleo de un predicado no verbal (PNV), como en (12).

S

(11) a. x-ø-el ulo [le sib']

CP-B3SG-salir DIR DET humo

'salió el humo'

○

b. $x-\varnothing-q a-q^{\prime}$ ol $\quad[l e$ kotz'i'j]

CP-B3SG-A1PL-cortar DET flor

'cortamos las flores'

A

c. m-at-u-ti' [le tz' $\left.\mathbf{t z}^{\prime} \mathbf{i}^{\prime}{ }^{\prime}\right]$ !

IMP.NEG-B2SG-A3SG-morder DET perro

'que no te muerda el perro!'

PNV

(12) $\varnothing$ ali le $r$-a al

B3SG mujer DET A3s-hijo.mujer

'su hija es mujer' 
Para convertir un sustantivo en verbo se puede agregar el intransitivizador - $\mathrm{Vr}$ a la raíz, como en (13a), al igual que los adjetivos, o una vocal para transitivizarlo, como en (13b).

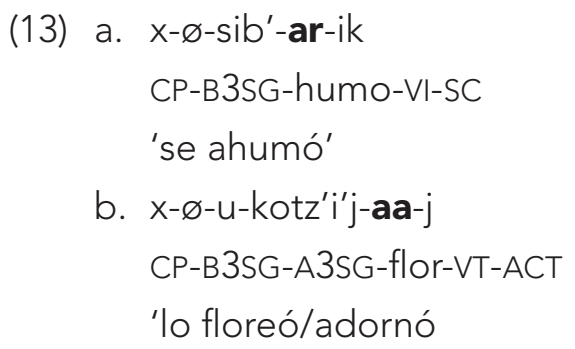

\subsection{Los posicionales}

Los posicionales son una clase de raíces muy productiva en idiomas mayas (England 2001, 2004, Santiz Gómez 2010, entre otros). En k'iche' se registran más de 300 raíces posicionales y pueden recibir hasta 14 afijos derivativos (Can Pixabaj y Sis Iboy, 2004). ${ }^{5}$ Las derivaciones más comunes son tres: el sufijo -VI para formar un estativo o predicado no verbal, el sufijo intransitivizador -e' que indica 'ponerse en la posición indicada por la raíz', y el sufijo transitivizador -(V)b'aa' o -V: que indica 'ponerlo en la posición indicada por la raíz. ${ }^{6}$

$\begin{array}{cllll}\text { raíz } & & \text { EST } & \text { verbo intransitivo } & \text { verbo transitivo } \\ \text { (14) } \text { tak' }^{\prime} \varnothing & \text { tak'-al-ik } & \text { x- } \varnothing \text {-tak'-e'-ik } & \text { x-ø-u-tak'-ab'aa' } \\ \text { parar } & \text { B3SG } & \text { parar-EST-SC } & \text { CP-B3SG-parar-VI-SC } & \text { CP-B3SG-A3SG-parar-VT } \\ \text { 'pararse' } & \text { 'está parado' } & \text { 'se paró' } & \text { 'lo paró' }\end{array}$

Algunas de estas raíces (aproximadamente 10\%) comparten morfología o rasgos con las raíces posicionales y con verbos transitivos radicales, como en el ejemplo (15), en donde la raíz toma las derivaciones de PNV y verbo intransitivo que toman las raíces posicionales, pero no toma la derivación transitiva porque como verbo transitivo se comporta como un verbo transitivo radical, sin ninguna derivación.

\footnotetext{
5 Hay raíces que son ambivalentes: posicionales y verbales a la vez.

6 Kaufman usa una nomenclatura diferente para cada tipo, a saber: estativo para el predicado no verbal, asuntivo para la forma intransitiva y depositivo para la forma causativa (Kaufman 1995). Estoy utilizando términos diferentes para la segunda y tercera derivación que me parecen un poco más claros.
} 


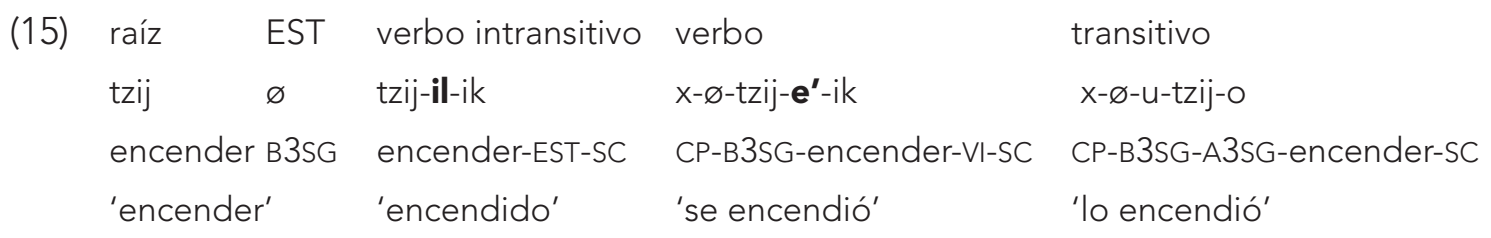

\subsection{Los verbos}

Los verbos llevan obligatoriamente marcas de tiempo/aspecto y persona gramatical, y pueden llevar sufijos de categoría que los identifica como transitivos o intransitivos.

Verbos intransitivos

(16) a. T/A+ absolutivo + raíz/base intransitiva + sufijo-categoría

b. x-at-war-ik COM-B2S-dormir-SC 'te dormiste.'

Los verbos transitivos pueden ser radicales o derivados. Los transitivos radicales tienen la estructura fonológica CVC y los derivados tienen estructura variada y más compleja, ya que se derivan de otras clases de palabras y siempre terminan en -j cuando el verbo está en voz activa. En (17a), el ejemplo es un verbo transitivo radical y en (17b) es derivado.

(17) a. T/A + absolutivo + ergativo + raíz/base transitiva + sufijo-categoría

b. X-at-u-chap-o

CP-B2SG-A3SG-agarrar-SC

'te agarró'

C. $\mathbf{x}$-at-u-q'aluu-j

CP-B3SG-A3SG-abrazar-ACT

'te abrazó'

\subsubsection{Derivaciones comunes de los verbos}

La derivación más relevante que toman los verbos intransitivos es el causativo -(ti) saa. El sufijo tiene la forma -tisaa cuando se agrega directamente a raíces intransitivas y tiene la forma -isaa o -saa (variación libre) cuando se agrega a otras bases 
intransitivas. La vocal de la última sílaba puede ser corta o prolongada; es prolongada cuando el verbo se encuentra al final de la frase entonacional o cuando no hay más sufijos después del causativo, de lo contrario se realiza como vocal corta.

\section{(18) X-at-u-war-tisaa-j \\ CP-B2SG-A3SG-sleep-CAU-ACT \\ 'te adormeció'}

Los verbos transitivos toman dos derivaciones relevantes que son el pasivo y el antipasivo. Un verbo activo transitivo marca dos argumentos en el núcleo verbal, Agente y Objeto, como en (19a). En la voz pasiva, el A se demueve a una frase oblicua que puede o no ser explícita y el verbo se vuelve intransitivo por lo que lleva una sola marca de persona que es el juego B y corresponde al sujeto sintáctico del verbo, como en (19b).
a. x-at-u-chap
le
q'atb'altziij
CP-B2SG-A3SG-agarrar DET
autoridad

'te atrapó/agarró la autoridad'
b. x-at-chaap r-umal le q'atb'altziij
CP-B2SG-agarrar.PAS A3SG-SR.AGT DET autoridad
'fuiste agarrado/atrapado por la autoridad'

En la literatura se han descrito dos tipos de pasivo: el pasivo sintáctico y el pasivo léxico (López 1997 y Larsen 1988). . El sintáctico se marca con la prolongación de la vocal de la raíz en verbos transitivos radicales (VTR), como en (19), y en verbos transitivos derivados con el sufijo $-x$, como en (20b). Un agente oblicuo es posible con ambos pasivos cuando el agente es tercera persona, como en (19b) y (20b).
(20)
a. le altomaab' $x-\varnothing-k i-q^{\prime}$ alu-j le nee'
DET señoritas CP-B3SG-A3PL-abrazar-ACT DET bebé
'el bebé fue abrazado por las señoritas'
b. $x-\varnothing-q^{\prime}$ alu-x k-umal le altomaab'
CP-B3SG-abrazar-PAS A3PL-SR.AGT DET señoritas
'(él) fue abrazado por las señoritas'

7 Cuando el agente oblicuo es primera o segunda solo se puede indicar cuando el pasivo es pasivo léxico, pero no se puede agregar cuando es pasivo sintáctico. 
El pasivo léxico se marca con el sufijo -Vtaj. Indica la finalización o terminación de un evento.

(21) a. x-at-chap-ataj-ik

CP-B2SG-agarrar-PAS-SC

'(finalmente) fuiste agarrado'

El antipasivo absolutivo y el enfoque agentivo se derivan con -ow y -on con verbos radicales y con $-n$ con verbos derivados. En este tipo de voz el verbo se intransitiviza y ya solo lleva la marca de una persona gramatical, el agente semántico. El paciente se puede expresar en una frase oblicua con el sustantivo relacional -eech o-e (variación libre).

(22)
a. are le q'atb'altziij $x$ - $\varnothing$-chap-ow aw-e
FOC DET autoridad CP-B3SG-agarrar-AP A2S-SR.AGT
'fue la autoridad quien te agarró/atrapó'
b. x-at-k'ayi-n-ik
CP-B2SG-vender-AP-SC
'vendiste'

En (22a) el ejemplo corresponde al enfoque agentivo; la FN de agente se encuentra en posición de foco y el paciente se encuentra en una frase oblicua. En (22b) el ejemplo corresponde al antipasivo absolutivo en donde el paciente no es mencionado.

Más adelante, en $\S 3$ veremos que la morfología pasiva y antipasiva se emplean para derivar el par incoativo en el tipo anticausativo; sin embargo, veremos que aquellos verbos con semántica incoativa no pueden recibir ni agente ni paciente oblicuo, como en los casos que hemos visto en esta sección, y por ello sostengo que son verbos incoativos.

\subsection{Metodología}

Para la realización de este estudio seguí los siguientes pasos:

- Primero, tomé como base principal el listado de 31 pares de verbos de Haspelmath (1993) de los cuales solo 22 funcionan en k'iche' (los verbos res- 
tantes no cumplen con la definición de la alternancia de pares de verbos incoativo-causativo). De este listado pude constatar que algunos de los verbos que participan en dicha alternancia vienen de posicionales y de verbos derivados con morfología causativa, pasiva y antipasiva.

- A continuación tomé la base de 300 raíces posicionales para identificar otros verbos que podrían participar en dicha alternancia. De aproximadamente 300 raíces posicionales, 21 participan en la alternancia de pares incoativo-causativo. ${ }^{8}$

- En la revisión de los datos fui agregando verbos que cumplen con la definición de los pares de verbos que participan en la alternancia de pares incoativo-causativo y en total obtuve 60 pares (véase apéndice).

- Finalmente, corroboré los datos en el campo, incluyendo las pruebas de restricción en cuanto a la adición o noción de un agente o paciente oblicuo.

\section{Los pares de verbos incoativo-causativos}

En esta sección primero voy a dar un resumen de los puntos principales que Haspelmath (1993) presenta y que son relevantes para este estudio; luego voy a discutir los datos del k'iche' con base en la propuesta de este autor (1993).

\subsection{Propuesta de Haspelmath}

Haspelmath (1993) aborda el tema de los pares de verbos incoativo-causativo considerando varios datos e indica que la dirección de la derivación no es universal, por ejemplo, en inglés el nombre de la ciencia puede ser la palabra básica y el científico la palabra derivada o a la inversa, como lo ilustran los ejemplos en (23).

8 Los datos que aquí incluyo son de SL. Los números que presento son aproximados y podrían variar, probablemente aumentar, porque se encuentran algunas variaciones en cuanto a los juicios de los hablantes respecto de la gramaticalidad e interpretación de estos como incoativos.

9 Hay dos aspectos que Haspelmath discute en su artículo que aquí no considero. El primero tiene que ver con los criterios para identificar las formas básicas, entre los que se encuentran (97): 1. fonología marcada, 2. dirección de neutralización, y 3. productividad. El segundo tiene que ver con la simplicidad conceptual como la base. En tal situación hace referencia a la correlación de la relación de la derivación básica y la relación formal y semántica que deben ser entendidas en términos cognitivos, siguiendo el principio de Givón (1991, en Haspelmath 1993). La correlación entre las relaciones básico-derivado formal y semántico debería entenderse en términos cognitivos como el principio de Givón. Las categorías que son cognitivamente marcadas tienden a ser estructuralmente marcadas. Así, los eventos que son más probables de ocurrir de manera espontánea estarán asociados con un estereotipo (o prototipo) conceptual de un evento espontáneo, al igual que eventos causados. Esto explicaría por qué algunos eventos tienen al incoativo como forma básica y otros al causativo. 
ciencia

(23)
a. physics 'física'
científico
physicist 'físico'
ciencia

b. linguist

'lingüista' científico

linguistics 'lingüística'

En (23a) se encuentra la forma no marcada. Es decir, se espera que el sustantivo que designa el oficio del científico se derive de la ciencia; sin embargo, en (23b) se revierte el orden porque del sustantivo que designa el oficio del científico se deriva el nombre de la ciencia y no a la inversa, y esto es a lo que Haspelmath llama formación inversa de palabras (reverse word formation). Esto es solo una muestra de que la dirección de la derivación no es universal porque aun en una misma lengua puede tomar diferente dirección.

Los puntos principales que discute Haspelmath, relevantes para este artículo, son: los tipos de alternancia o patrones de codificación, la definición semántica de los pares de verbos que participan en la alternancia incoativo-causativo, los criterios relevantes para diferenciarlos de otros verbos y las restricciones que excluyen posibles candidatos.

Haspelmath (1993) enuncia la definición semántica de un par de verbos con la alternancia incoativo-causativo como «un par de verbos que expresan la misma situación básica (generalmente un cambio de estado y muy raras veces algo en proceso)". ${ }^{10}$ Sigue indicando que el causativo incluye un participante, quien causa la situación y que generalmente es transitivo. Un incoativo, por otro lado, excluye un agente causante y presenta la situación con ocurrencia espontánea y generalmente se expresa léxicamente en un verbo intransitivo. Zavala Maldonado (2001) agrega que el incoativo se refiere a eventos o procesos que resultan en un cambio de estado, condición, posición o locación del único participante.

El miembro incoativo de un par verbal incoativo-causativo es semánticamente similar al pasivo de un causativo, pero la situación se concibe como ocurrida sin agente y de manera espontánea.

Haspelmath propone tres tipos principales de la alternancia incoativo-causativo.

I. Causativo: el verbo incoativo es la forma básica y el causativo es la forma derivada que puede o no tener derivación morfológica.

\footnotetext{
${ }^{10}$ «it is a pair of verbs which express the same basic situation (generally a change of state, more rarely a goin-on", and differ only in that the causative verb meaning includes an agent participant who causes the situation, whereas in the inchoative verb meaning excludes a causing agent and presents the situation as occurring spontaneously (traducción mía) (Haspelmath 1993:90).
} 
II. Anticausativo: el verbo causativo es la forma básica, el incoativo es la forma derivada. Puede registrar cambios en su morfología.

III. Alternancias no directas (u opuestas): ni el incoativo es derivado del causativo ni viceversa. Aquí hay tres subtipos:

a) lábil: el mismo verbo es usado para los sentidos incoativo y causativo.

b) equipolente: ambos son derivados de la misma base (ninguno se deriva del otro).

c) supletivo: se emplean diferentes bases, una para la forma incoativa y otra para la causativa.

Además, Haspelmath (1993:92) indica cuáles son las restricciones semánticas sobre los pares incoativo-causativo, entre las que menciona que: a) no toda situación ocurre en la alternancia incoativo-causativo y b) la situación básica debe ser un cambio de estado o el cambio en proceso. Luego indica que hay tres situaciones que se excluyen: i) un estado no puede ser el miembro incoativo de una alternancia incoativo-causativo, ii) una acción que no expresa un cambio de estado no puede ser el miembro incoativo en la alternancia, y iii) verbos intransitivos agentivos, como 'hablar', no pueden ser el miembro incoativo en la alternancia. c) La condición semántica específica más importante en el estudio de los pares de verbos incoativo-causativo es la ausencia de un significado con orientación de agente.

Finalmente indica que hay una escala de probabilidad de incremento de ocurrencia espontánea (Haspelmath 1993:105).

(24) lavar $\rightarrow$ cerrar $\rightarrow$ derretir $\rightarrow$ reírse

Los verbos en la izquierda de la escala tienen menos probabilidad de ocurrir de manera espontánea, mientras que los de la derecha sí la tienen.

En la siguiente sección paso a los datos del k'iche' enfatizando los puntos relevantes de Haspelmath: primero los tipos o patrones de alternancia de pares incoativo-causativo, luego la definición semántica y finalmente los criterios o restricciones semánticas sobre dichos pares.

\subsection{La alternancia de los pares incoativo-causativo en k'iche'}

En k'iche' hay tres tipos de alternancia incoativo-causativo con base en la clasificación de Haspelmath (1993): anticausativo, causativo (directo) y equipolente (no 
directo). Abajo describo el comportamiento de cada tipo, el cual incluye cambios morfosintácticos como la adición de sufijos derivativos y argumentos oblicuos. Sin embargo, antes de discutir cada tipo de alternancia es importante aclarar que el par incoativo de la alternancia incoativo-causativo en k'iche', siguiendo a Haspelmath (1993), debe indicar un cambio de estado, debe ser espontáneo y no incluir un causante externo.

\subsubsection{Anticausativo}

En el tipo anticausativo el verbo básico es el causativo y el incoativo es el derivado. El causativo es un verbo transitivo que puede ser radical como en (25-26) o derivado como en (27). En todos los casos, el incoativo se deriva con un sufijo que puede ser la prolongación vocálica como en (25), -Vtaj, como en (26) y -n, como en (27).

causativo

(25) a. $x-\varnothing-u-k^{\prime} a t-o$

CP-B3SG-A3S-quemar-SC

'lo quemó'

b. $x$-u-tzaq-o

CP-B3SG-A3SG-botar-SC

'lo botó'

(26) a. x-ø-u-yak-o

CP-B3SG-A3SG-apagar-SC

'lo leventó'

b. $x-\varnothing-u-j a q-o$

CP-B3SG-A3SG-abrir-SC

'lo abrió'

(27) a. $\quad x-\varnothing-u-j a b ' u u-j$

CP-B3SG-A3SG-dispersar-SC

'lo dispersó'

b. $x-\varnothing-u-k o t z^{\prime} i^{\prime} j a a-j$

CP-B3SG-A3SG- florear.adornar-ACT

'lo adornó/le puso flores' incoativo

$x-\varnothing-k^{\prime}$ aat-ik

CP-B3SG-quemar.PAS-SC

'se quemó'

x-tzaaq-ik

CP-B3SG-caer.PAS-SC

'se cayó'

x-ø-yak-ataj-ik

CP-B3SG-apagar.PAS-SC

'se levantó'

$x$ - $\varnothing$-jaq-ataj-ik

CP-B3SG-abrir.PAS-SC

'se abrió'

$\mathrm{x}-\varnothing$-jab'u-n-ik

CP-B3SG-dispersar.AP-SC

'se dispersó'

x-ø-kotz'i'ja-n-ik

CP-B3SG-florear-AP-SC

'floreó (una flor)' 
Los sufijos -V: y -Vtaj son los mismos sufijos que derivan el pasivo (25-26), como en (19b y 20b), en donde derivan el pasivo claramente. Sin embargo, en (25-26) sostengo que derivan el incoativo. El sufijo -n en (27) es el mismo que deriva el antipasivo, como en (22a); pero en (27) argumento que está derivando el incoativo. Así, los datos presentados hacen una fuerte sugerencia en cuanto a la derivación del incoativo. Por un lado, el incoativo se deriva con morfología pasiva y por el otro con antipasiva. Por esta razón hago una subclasificación de pasivo-incoativo y antipasivo-incoativo.

\subsubsection{Pasivo-incoativo}

En el primer grupo de anticausativos en (25) el incoativo se forma por medio de la prolongación de la vocal de la raíz que es igual a la formación del pasivo con verbos transitivos radicales. Es decir, estos verbos incoativos tienen la forma de un pasivo. Como Haspelmath indica, una de las características principales del verbo incoativo es que no incluye un causante externo. En este caso es necesario mostrar que el verbo no puede incluir un causante externo y la manera de hacerlo es agregando un agente oblicuo, como en (28b), en donde se puede ver que la oración resulta agramatical. Entonces se puede decir que la prueba de la adición de un agente oblicuo muestra que en (28a) no puede haber un causante externo y por tal razón lo que se tiene es un verbo incoativo con forma pasiva.

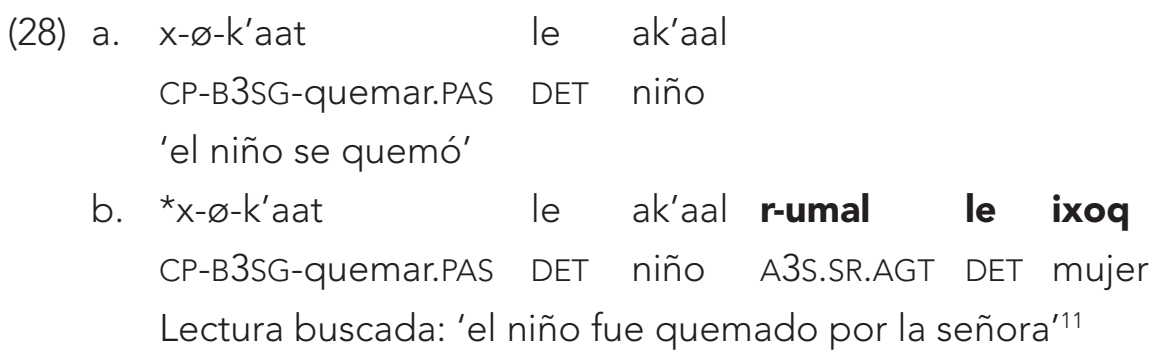

Sin embargo, en otros casos puede haber ambigüedad entre el incoativo que no incluye agente y el pasivo que sí puede incluir un agente oblicuo. Los verbos incoativos en (26) se derivan con el sufijo -Vtaj que deriva el pasivo léxico. En un solo caso, que es el verbo yak-ataj 'levantarse', no se puede agregar un agente

11 Para obtener la lectura buscada es necesario agregar el morfema causativo a la base y después agregar el sufijo pasivo como en el siguiente ejemplo:
$\times$ - $\varnothing-k^{\prime}$ at-sa-x
le
ak'aal r-umal
le ixoq
CP-B3SG-quemar.CAU-PAS DET niño A3S.SR.AGT DET mujer
'el niño fue quemado por la señora.' 
oblicuo; es decir, solo en este caso, para saber si el verbo es incoativo o no, se recurre a la prueba de adición del agente oblicuo o simplemente al contexto.

En (29) se muestra un ejemplo de este grupo. En (29a) se encuentra la forma sin agente oblicuo, la cual se puede interpretar como incoativo (la puerta se abrió por sí sola o la puerta fue abierta por alguien no mencionado). En (29b) se presenta la forma con la adición del agente oblicuo en donde ya no hay cabida para una interpretación incoativa porque no solo existe la noción de la existencia de un agente externo, sino que dicho agente está expresado.
a. X-ø-jaq-ataj le uchi ja
CP-B3SG-abrir-PAS DET puerta
'se abrió la puerta'/la puerta fue abierta)'
b. x-ø-jaq-ataj le uchija (r-umal le ixoq)
CP-B3SG-abrir-PAS DET puerta A3S.SR.AGT DET mujer 'se abrió la puerta'/la puerta fue abierta (por la señora)'

\subsubsection{Antipasivo-incoativo}

Los ejemplos en (27) corresponden al segundo grupo de verbos del tipo anticausativo. Estos verbos se derivan con el sufijo - $n$ que deriva verbos antipasivos y el enfoque agentivo. ${ }^{12}$ En general, un antipasivo en $k^{\prime} i c h e^{\prime}$ es un verbo intransitivo que únicamente tiene marca del JB como sujeto sintáctico y agente semántico. El paciente se puede agregar en una frase oblicua, como en (30b).
(30) a. le ala
$\mathrm{x}-\varnothing-\mathrm{u}-\mathrm{k}^{\prime} \mathrm{am}$
le ali
DET muchacho CP-B3SG-A3SG-recibir.aceptar
DET muchacha
'el muchacho aceptó a la muchacha'
b. are le ala
$x-\varnothing-k^{\prime} a m-o w$
r-e
le ali
FOC DET muchacho CP-B3SG-recibir.aceptar-AP A3SG-SR.PAC DET muchacha
'fue el muchacho quien aceptó a la muchacha'

\footnotetext{
12 El enfoque agentivo puede tener una estructura más complicada que lo que aquí se presenta. Bajo ciertas condiciones y en variantes específicas del k'iche', el enfoque agentivo, además de tener el agente en posición de foco, el verbo concuerda con el paciente y no con el agente y el paciente puede mencionarse en una frase oblicua o sin la frase oblicua (para más detalles véase Mondloch 1981). En este estudio se presenta su forma sintáctica más básica en donde el agente aparece en primera posición, el verbo tiene la marca de agente y el paciente puede encontrarse en una frase oblicua.
} 
Ahora, voy a mostrar que el grupo de ejemplos en (31) son verbos incoativos y debido a que tienen morfología antipasiva los voy a contrastar con la estructura sintáctica de un verbo antipasivo (que claramente tiene un agente o causante externo), con atención al significado y la adición de un paciente oblicuo. En (31) incluyo el listado de ejemplos que ya presenté en (27). Primero, semánticamente, todos los ejemplos en (31) tienen un paciente semántico en vez de un agente semántico, como en el caso de los antipasivos. En contraste, el significado del verbo en (31b) 'aceptó' que es el agente semántico y el significado del verbo en (31a) 'explotó' en donde lo que explota no es el agente sino el paciente. Esa es la primera prueba que muestra que los verbos en (27 y 31) son incoativos y no antipasivos.
(31) a. paq'i-n 'explotar'
b. jab'u-n 'dispersarse'
c. kotz'i'ja-n 'florear'
d. raqi-n 'rajarse'

Segundo, si los verbos en (31) fueran antipasivos tendrían la posibilidad de agregar un paciente oblicuo. Sin embargo, este no es posible por dos razones estrechamente relacionadas: i) un antipasivo tiene un agente marcado en el núcleo verbal, pero ya vimos que el sujeto de los verbos en (31) son pacientes y no agentes semánticos; ii) un antipasivo tiene la posibilidad de agregar un paciente oblicuo; sin embargo, esto no puede hacerse con los ejemplos en (31). La razón es que el verbo ya tiene un paciente en el núcleo verbal, de manera que no se puede agregar otro. Esta es una restricción no solo semántica sino también sintáctica. En (32a) el nominal le ja 'la casa' es el sujeto/paciente del verbo xraqin. En (32b) se le ha agregado lo que podría ser un paciente oblicuo, pero resulta completamente agramatical, no se puede obtener ninguna lectura gramatical.
a. $x-\varnothing-$ raqi-n
le ja
COM-B3S-rajar-AP DET casa
'la casa se rajó'
b. *x-ø-raqi-n le ja
COM-B3S-rajar-AP DET casa
r-eech
le uleew
*'la casa se rajó a la tierra' 
Una manera de obtener una oración gramatical de (32b) es reordenando los elementos de la oración como en (33). En (33) le ja 'la casa' sigue siendo el sujeto del verbo, pero su función semántica cambia de paciente a agente y el elemento oblicuo sí se interpreta como el paciente. Sin embargo, en (33) el verbo ya no tiene una interpretación incoativa. El verbo es un antipasivo normal en donde hay un agente y un paciente sin que el evento sea espontáneo.

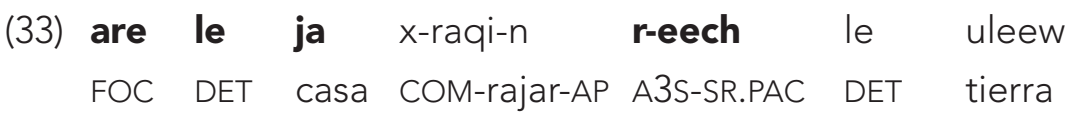

'fué la casa que rajó la tierra'

A partir de los datos presentados se puede ver que de los verbos transitivos radicales y los incoativos se derivan con morfología pasiva (25-26), y que de los verbos transitivos derivados se derivan incoativos con morfología antipasiva (27 y 31). Esta es solo una tendencia, ya que se encuentran algunas excepciones, por ejemplo, en (34) el verbo es transitivo derivado, pero emplea el sufijo pasivo -taj para derivar del incoativo.

(34)

x-ø-u-kunaa-j
CP-B3SG-A3SG-curar-SC
'lo curó'

\author{
$\mathrm{X}-\varnothing-\mathrm{kuna}$-taj-ik \\ CP-B3SG-curar.PAS-SC \\ 'se curó'
}

\subsubsection{Causativo}

En la alternancia causativa el verbo básico es el incoativo y el causativo es derivado. El incoativo puede ser una raíz o una base transitiva y el causativo es derivado con el sufijo -(ti)saa y uno con -ii. Lo relevante aquí es la forma del incoativo. El incoativo puede venir de varias raíces como de verbos intransitivos y adjetivos, como se presenta en los ejemplos de abajo. En (35) la forma incoativa viene de verbos intransitivos y la forma causativa toma el sufijo -(ti)saa. El verbo con el sufijo causativo -isaa, -isa se le trata como un verbo transitivo derivado debido a que recibe el sufijo -j, como cualquier verbo transitivo derivado. 


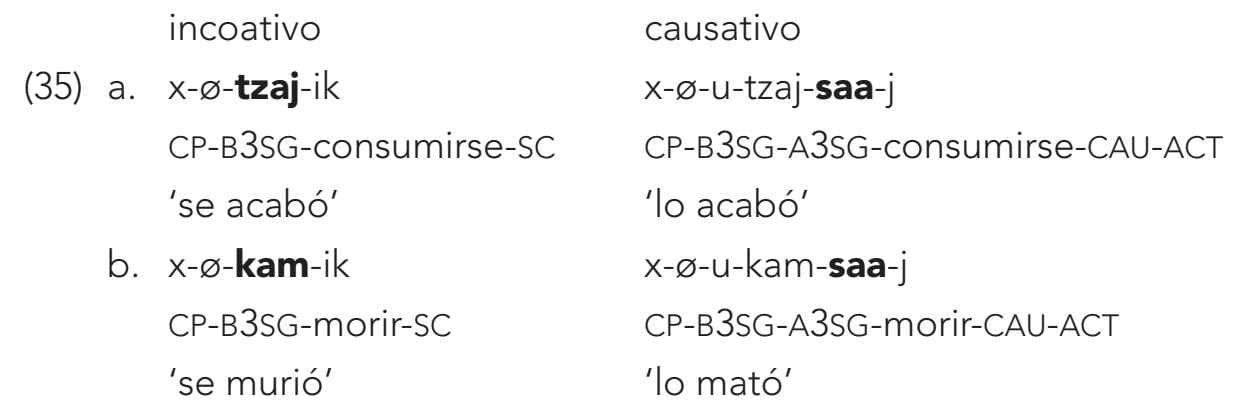

El ejemplo en (36a) tiene la raíz poq' 'hervir' y el sufijo -ow para convertirlo en un verbo intransitivo. El ejemplo en (36b) es una raíz intransitiva.

(36) a. X-ø-poq'ow-ik

CP-B3SG-hervir-SC

'hirvió'

b. $x$ - $\varnothing$-spoj-ik

CP-B3SG-hincharse-SC

'se hinchó'

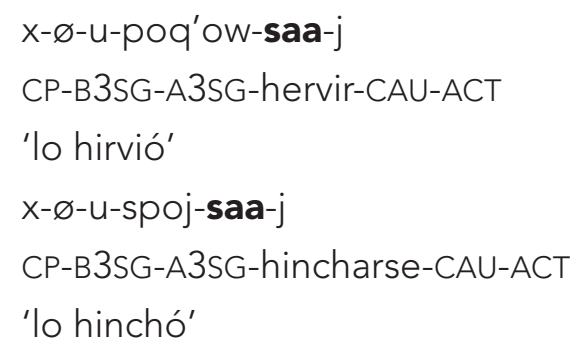

En (37-38) los verbos incoativos tienen bases intransitivas derivadas. Dichas bases se derivan de adjetivos por medio de dos tipos de sufijos. En (37) las bases intransitivas se derivan con el sufijo -(V)ry en (38) con el sufijo-ob'. El par transitivo se forma agregando el sufijo -(it)saa a esta base.

(37)
a. $\mathbf{x - \varnothing - j a ' - r - i k ~}$
CP-B3SG-agua-VI-SC
'se deshieló/aguó'
b. $x-\varnothing-k o o w-i r-i k$
CP-B3SG-duro-VI-SC
'endureció'

(38) a. X-ø-ri'j-ob'-ik

CP-B3SG-viejo-VI-SC

'maduró/envejeció'

b. $x-\varnothing-t z ' i l-o b^{\prime}-i k$

CP-B3SG-sucio-VI-SC

'se ensució'

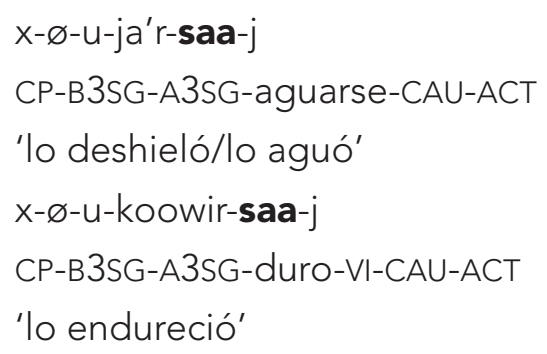

'lo endureció'

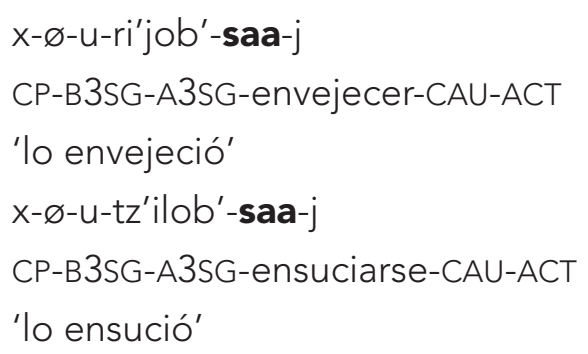


Nótese que en todos estos casos el verbo incoativo indica un cambio de estado, es espontáneo y no incluye un agente externo.

\subsubsection{Equipolente (no directo)}

Finalmente, de los tres subtipos del tipo 'no directo' sólo se han encontrado datos para el equipolente en k'iche'. En el equipolente tanto el incoativo como el causativo vienen de la misma raíz pero ninguno se deriva del otro. En k'iche' los ejemplos de este subtipo vienen de raíces posicionales o posicionales/transitivas. El incoativo se deriva con - $e^{\prime}$ y el causativo con el sufijo $-(V) b^{\prime} a a^{\prime} /-V_{\text {: }}$, como en (39 y 40) respectivamente. Cuando la raíz es ambivalente, posicional-verbal, se usa - $e^{\prime}$ para el incoativo y no hay sufijo derivativo para el causativo porque es un verbo transitivo radical, como en (41). ${ }^{13}$
incoativo
causativo
a. $x$ - $\varnothing$-tan- $\mathbf{e}^{\prime}$-ik
CP-B3SG-suspender-VI-SC
'se suspendió'
(40) a. x-ø-tz'ap-e'-ik
CP-B3SG-cerrar-VI-SC
'se cerró'
b. $\mathrm{x}-\varnothing-\mathbf{u}-\tan -\mathbf{a b} \mathbf{b a}^{\prime}$
CP-B3SG-A3SG-suspender-VT
'lo suspendió'
b. $\mathbf{x}-\varnothing-\mathbf{u}-\mathrm{tz}^{\prime} \mathrm{ap}-\mathbf{i i}-\mathrm{j}$
CP-B3SG-A3SG-cerrar-VT-ACT
'lo cerró'
(41) a. x-ø-tzij-e'-ik
CP-B3SG-encender-VI-SC
'se encendió'

b. $x-\varnothing-u-t z i j-o$

CP-B3SG-A3SG-encender-SC

'lo encendió'

El verbo incoativo en el tipo equipolente se da de manera espontánea, no incluye un causante externo y el sujeto corresponde al paciente semántico.

\section{Resumen}

En las siguientes tablas presento un resumen sobre la alternancia de pares incoativo-causativo en k'iche' y un resumen sobre la cantidad de pares encontrados.

13 El miembro incoativo del par incoativo-causativo se ha reportado como tal en otros idiomas mayas, por ejemplo, en tseltal, por Sántiz Gómez (2010) y Polian (2011). 
En la Tabla 2, lo que aparece en negritas corresponde a la forma básica del par, si lo hay.

Tabla 2. Tipos de alternancia de pares incoativo-causativo

\begin{tabular}{lll}
\hline tipos de alternancia & forma incoativa & forma causativa \\
anticausativo & $-V_{t a j},-V_{:},-n$ & base transitiva \\
causativo & base intransitiva & - tisaa, $-V_{:}$ \\
equipolente & $-e^{\prime}$ & $-V^{\prime} b^{\prime} a a^{\prime},-V_{i, \varnothing}$ \\
\hline
\end{tabular}

Fuente: elaboración propia

En la Tabla 3 presento las cantidades de ejemplos encontrados para cada patrón de codificación de los pares de verbos incoativo-causativo.

Tabla 3. Número de pares incoativo-causativo encontrados

\begin{tabular}{cc}
\hline Patrón de codificación & Cantidad de verbos encontrados \\
\hline anticausativo & 18 \\
causativo & 21 \\
equipolente & 21 \\
Total & 60 \\
\hline
\end{tabular}

Fuente: elaboración propia

Vale la pena mencionar que el tipo 'anticausativo' sí se encuentra en k'iche' y que se encontraron 18 de los 60 casos. Esto apoyaría la propuesta de Haspelmath (1993) en decir que esta alternancia no puede ser limitada a un grupo de verbos con cierta coherencia semántica como lo indica Marantz. Sin embargo, en la muestra de Haspelmath este patrón solo se encontró en lenguas europeas, pero no en otras lenguas de otras familias. Así, Haspelmath concluye preguntando si el parámetro tipológico de preferencia por el anticausativo vs. causativo está conectado con otros factores de la lengua. Las correlaciones que encontró son dos: (i) areal, las lenguas de su muestra que tienen preferencia por la alternancia anticausativa se hablan en Europa y (ii) relación genética, todas están relacionadas genéticamente. 
El k'iche' no prefiere este tipo pero sí tiene la alternancia anticausativa; sin embargo, las razones que Haspelmath ofrece para las lenguas de su muestra obviamente no explican por qué el k'iche' tiene esta alternancia porque ni está en área europea ni se relaciona genéticamente con ninguna familia europea. Entonces, si se postula que este tipo no es común, aún no se sabe por qué el k'iche' lo tiene.

\section{Conclusiones}

El k'iche' tiene los tres patrones de codificación de alternancia de los pares incoativo-causativo que Haspelmath (1993) propone: anticausativo, causativo y equipolente (del tipo no directo). En cierta forma existe un grado de predictibilidad de cada patrón, por ejemplo, el patrón causativo ocurre cuando el verbo básico es un verbo intransitivo radical o derivado de adjetivos o sustantivos; el patrón anticausativo generalmente ocurre cuando el verbo derivado toma morfología pasiva o antipasiva, y el patrón equipolente generalmente se encuentra cuando la raíz que comparten el par de verbos es una raíz posicional.

En el patrón anticausativo se emplea la morfología pasiva y antipasiva para formar el par incoativo. En estos casos es agramatical agregar un agente o paciente oblicuo como se espera con verbos pasivos y antipasivos canónicos. Cuando el incoativo se forma con el -Vtaj existe ambigüedad entre incoativo y pasivo. La ambigüedad se puede resolver sintáctica o contextualmente. Este patrón es importante en dos sentidos. Primero, el uso de la morfología pasiva y antipasiva para formar el par incoativo es relevante porque es un caso en que no hay correlatos entre la morfología y la sintaxis; prueba de ello es que no puede agregarse un agente oblicuo para la forma pasiva, situación que reafirma el sentido incoativo del verbo, así como la agramaticalidad de la adición de un paciente oblicuo para la forma antipasiva. Esto es importante porque este uso del pasivo y antipasivo no había sido discutido, por lo menos para el k'iche'. Segundo, tipológicamente la existencia del tipo anticausativo se encuentra en debate. Por un lado, Marantz (en Haspelmath 1993) indica que este tipo ocurre con un número limitado de verbos; por el otro, Haspelmath muestra que en lenguas europeas el número de casos no es muy limitado, pero existe solo en lenguas genéticamente relacionadas. El k'iche' muestra que el tipo anticausativo existe en esta lengua y que esto cuestiona lo que indican los autores mencionados. Entonces, el k'iche' hace un aporte tipológico muy importante. Con este resultado se podría decir 
que la alternancia 'anticausativa' no es muy común, pero que puede ocurrir en ciertas familias de lenguas como las europeas y las mayas.

El patrón equipolente también es relevante porque aquí se usan las raíces posicionales de manera productiva. Las raíces posicionales son una clase común en las lenguas mayas; sin embargo, no hay estudios detallados sobre sus contextos de uso y este sería solo uno de ellos. Finalmente, de los tres patrones, el causativo es el menos marcado, la forma básica es un verbo intransitivo radical o derivado principalmente de un adjetivo o sustantivo y la forma causativa o derivada se forma con el causativo morfológico -(ti)saa o $\mathrm{V}$.

El resultado de este estudio podría generalizarse a otras lenguas, especialmente a las lenguas del grupo k'iche'; sin embargo el uso de los posicionales pasivos y antipasivos podría ser generalizado a otras lenguas.

\section{Referencias bibliográficas}

Can Pixabaj, Telma y Nikte' María Juliana Sis Iboy, 2004, « Contextualizando posicionales K'ichee'-Achi'», Lengua y mantenimiento cultural en Mesoamérica: Un Simposio, The University of Texas at Austin.

Can Pixabaj, Telma, 2007, «El pasivo completivo en K'ichee'», ponencia presentada en el CILLA III, Austin Texas,

England, Nora C., 2001, Introducción a la lingüística maya, Guatemala, Cholsamaj.

_ 2004, «Entrando y saliendo de una posición: Palabras Afectivas en Mam», ponencia presentada en Lengua y Mantenimiento Cultural en Mesoamérica: Un simposio, Center of Indigenous Languages of Latin America. Austin, Universidad de Texas.

Haspelmath, Martin, 1993, «More on the typology of inchoative/causative verb alternations», en Causatives and transitivity, compilado por Bernard Comrie and Maria Polinsky, Amsterdam, John Benjamins.

Kaufman, Terrence, 1995, Positionals in Meje-Zoquean, Pensilvania, University of Pittsburg, (ms.).

Larsen, Thomas Walter, 1988, Manifestations of Ergativity in Quiché Grammar, tesis doctoral inédita, Berkeley, Universidad de California.

López Ixcoy, Candelaria Dominga (Saqijix), 1997, Ri Ukemiik ri K'ichee' Chii': Gramática K'ichee', Guatemala, Cholsamaj.

Mondloch, James Lorin, 1981, Voice in Quiché-Maya, disertación doctoral, Albany, State University of New York.

Polian, Gilles, 2013, Gramática del tseltal de Oxchuc, México, CIESAS (Publicaciones de la Casa Chata). 
Richards, Michael, 2003 Atlas Lingüístico de Guatemala, Guatemala, Universidad Rafael Landivar.

Sántiz Gómez, Roberto, 2010, Raíces posicionales en tseltal de Oxchuc, tesis de maestría inédita, México, CIESAS.

Zavala Maldonado, Roberto, 2001, "Olutec causatives and applicatives», en Masayoshi Shibatani (ed.), The grammar of causation and interpersonal manipulation, Ámsterdam y Filadelfia, Jonh Benjamins, pp. 245-249.

\section{Apéndice}

El siguiente cuadro contiene los pares de verbos identificados y analizados en el presente estudio. La columna del k'iche' contiene únicamente las raíces o bases verbales.

Tabla 4. Verbos que participan en la alternancia de pares incoativo-causativo

\begin{tabular}{|c|c|c|c|}
\hline Cantidad & Patrón & Español & K'iche' \\
\hline 1 & equipolente & cerrarlo/cerrarse & tz'ape'/tz'apii \\
\hline 2 & equipolente & despertarlo/despertarse & k'asuu/k'astaj \\
\hline 3 & equipolente & detenerlo/detenerse & tak'e'/tak'ab'aa' \\
\hline 4 & equipolente & posar (agua) & $\begin{array}{l}\text { cheme'/ } \\
\text { chemeb'aa' }\end{array}$ \\
\hline 5 & equipolente & mojarse/mojarlo & $\begin{array}{l}\text { ch'aqe' } \\
\text { ch'aqab'aa' }\end{array}$ \\
\hline 6 & equipolente & Trabarse & $\begin{array}{l}\text { ch'epe' } \\
\text { ch'epeb'aa' }\end{array}$ \\
\hline 7 & equipolente & amarrar duro & jat'e'/jat'ii \\
\hline 8 & equipolente & Torcer & jech'e'/jech'eb'aa' \\
\hline 9 & equipolente & sacar llamas (abundantes) & jine'/jinib'aa' \\
\hline 10 & equipolente & ponerse Redondo & kete'/keteb'aa' \\
\hline 11 & equipolente & ponerse curvado & kote'/kotob'aa' \\
\hline 12 & equipolente & empaparse (más que ch'aqe'ik) & rub'e'/rub'ub'aa' \\
\hline 13 & equipolente & Cesar & tane'/tanab'aa' \\
\hline 14 & equipolente & ponerse en forma de bolita & t'ore'/t'orob'aa' \\
\hline 15 & equipolente & $\begin{array}{l}\text { ponerse boludo (grande } \\
\text { en volumen) }\end{array}$ & t'oye'/t'oyob'aa' \\
\hline 16 & equipolente & hundir/abollar & yot'e'/yot'ob'aa' \\
\hline
\end{tabular}




\begin{tabular}{|c|c|c|c|}
\hline Cantidad & Patrón & Español & K'iche' \\
\hline 17 & equipolente & cerrar los ojos & yupe'/yupub'aa' \\
\hline 18 & equipolente & sacar la punta (algo puntiagudo) & jise'/jisib'aa' \\
\hline 19 & equipolente & $\begin{array}{l}\text { resaltar o mostrar (algo como } \\
\text { los dientes) }\end{array}$ & kitze'/kitzib'aa' \\
\hline 20 & equipolente & caer o doblar (orejas de un perro) & luq'e'/luq'ub'aa' \\
\hline 21 & equipolente & Enderezarse & suk'e'/suk'ub'aa' \\
\hline 1 & causativo & quemarlo/quemarse & k'at/k'aat \\
\hline 2 & causativo & abrirlo/abrirse & jaq/jaqataj \\
\hline 3 & causativo & Juntar & mulii/mulitaj \\
\hline 4 & causativo & Dispersar & jab'uu/jab'un \\
\hline 5 & causativo & Cambiar & jal/jalataj \\
\hline 6 & causativo & Destruir & wilii/wilitaj \\
\hline 7 & causativo & perderlo/perderse & tzaq/tzaaq \\
\hline 8 & causativo & levantarlo/levantarse & yak/xyakataj \\
\hline 9 & causativo & terminarlo/terminarse & $k^{\prime}$ is/k'iis \\
\hline 10 & causativo & doblarlo/doblarse & q'och/q'oche' \\
\hline 11 & causativo & Rodar & b'alkatii/b'alkatin \\
\hline 12 & causativo & Mejorar & suk'maa/suk'mataj \\
\hline 13 & causativo & rajar/rajarse (cosas) & paq'/paq'ataj \\
\hline 14 & causativo & adornar/florear & kotz'i'jaa/kotz'i'jan \\
\hline 15 & causativo & rajar/rajarse (plans) & raqii/raqin \\
\hline 16 & causativo & Apagar & chup/chuputaj \\
\hline 17 & causativo & poner en forma cilíndrica & xb'al/xb'ale' \\
\hline 18 & causativo & pegar & xnak'/xnak'e' \\
\hline 19 & causativo & prender.encender & xtzij/xtzije' \\
\hline 20 & causativo & ponerse con llave & $x j u^{\prime} / x j u^{\prime} e^{\prime}$ \\
\hline 21 & causativo & partir/partirse & pir/piritaj \\
\hline 1 & anticausativo & quebrarse/quebrarlo & pax/paxii \\
\hline 2 & anticausativo & morir/matar & kam/kamsaa \\
\hline 3 & anticausativo & derretirse/derretirlo & ja'r/ja'rsaa \\
\hline 4 & anticausativo & hervirse/hervirlo & paq'ow/paq'owsaa \\
\hline 5 & anticausativo & congelarse/congelarlo & kowir/kowirsaa \\
\hline 6 & anticausativo & Ilenarse/llenarlo & nooj/nojsaa \\
\hline 7 & anticausativo & secarse/secarlo & $\begin{array}{l}\text { chaqi'jar/ } \\
\text { chaqi'jarsaa }\end{array}$ \\
\hline 8 & anticausativo & ahogar/ahogarse & jiq'/jiq'saa \\
\hline
\end{tabular}




\begin{tabular}{|l|l|l|l|}
\hline Cantidad & Patrón & Español & K'iche' \\
\hline 9 & anticausativo & quemarse/quemarlo & qu'w/qu'wsaa \\
\hline 10 & anticausativo & pudrirse/pudrirlo & q'ay/q'aysaa \\
\hline 11 & anticausativo & hincharse/hincharlo & spoj/spojsaa \\
\hline 12 & anticausativo & secarse/secarlo & $\begin{array}{l}\text { chaqi'jar } \\
\text { chaqi'jarsaa }\end{array}$ \\
\hline 13 & anticausativo & ahumarse/ahumarlo & sib'ar/sib'arsaa \\
\hline 14 & anticausativo & envejece/envejecerlo (animado) & ri'jar/ri'jarsaa \\
\hline 15 & anticausativo & ensuciarse/ensuciarlo & tz'ilob'/tz'ilob'saa \\
\hline 16 & anticausativo & enfriarse/enfriarlo & jorob'/jorob'saa \\
\hline 17 & anticausativo & calentarse/calentarlo & k'atanob'ir/ \\
\hline 18 & & & k'atanob'irsaa \\
\hline total $=60$ & & anticausativo & crecer/criarlo $/ k^{\prime}$ iytisaa \\
\hline & & & \\
\hline
\end{tabular}

Fuente: elaboración propia 